Portland State University

PDXScholar

$5-1-1980$

\title{
Polarization characteristics of dye-laser amplifiers I. Unidirectional molecular distributions
}

Lee W. Casperson

Portland State University

Kendall C. Reyzer

Follow this and additional works at: https://pdxscholar.library.pdx.edu/ece_fac

Part of the Electrical and Computer Engineering Commons

Let us know how access to this document benefits you.

\section{Citation Details}

Casperson, L. W., Reyzer, K. C. (1980). Polarization characteristics of dye-laser amplifiers I. Unidirectional molecular distributions. Journal of Applied Physics, 51 (12), 6075-6082.

This Article is brought to you for free and open access. It has been accepted for inclusion in Electrical and Computer Engineering Faculty Publications and Presentations by an authorized administrator of PDXScholar. Please contact us if we can make this document more accessible: pdxscholar@pdx.edu. 


\title{
Polarization characteristics of dye-laser amplifiers I. Unidirectional molecular distributions
}

\author{
Kendall C. Reyzer and Lee W. Casperson \\ School of Engineering and Applied Science, University of California, Los Angeles, California 90024
}

(Received 15 April 1980; accepted for publication 20 May 1980)

\begin{abstract}
Many practical laser amplifiers exhibit anisotropic gain due to polarization of the pumping fields or to a fixed preferential alignment of the active dipoles. Several specific causes and consequences of gain anisotropy are discussed in detail. In the analysis, the emphasis is placed on dye-laser systems including arbitrary amplitudes, phases, and polarizations of the pump and signal fields. Analytical results are presented for a unidrectional molecular distribution, and it is found that the polarization states of the pump and signal fields change with distance in the amplifier.
\end{abstract}

PACS numbers: 42.55.Mv, 42.10.Nh

\section{INTRODUCTION}

Lasers that operate with isotropic pumping mechanisms such as flashlamps or electric discharges are normally expected to produce an output beam that is unpolarized. Polarized outputs only occur when some anisotropy is introduced into the resonator or lasing medium. These anisotropies are present, for example, in solid-state lasers when the host crystal is biaxial or uniaxial, in gas lasers when a brewster window is used on the laser tube, or in lasers where the pumping mechanism itself is anisotropic. The purpose of this study is to investigate in detail the polarization characteristics of anisotropically pumped laser amplifiers with emphasis on dye lasers. Dye systems provide one of the best known examples of laser amplifiers in which the gain may be strongly anisotropic. The effects of this anisotropy on the optical properties of a laser amplifier have not been previously considered in detail.

The initial discovery of the dye laser was made using another laser as the pumping source. The pump laser was linearly polarized and thus the excitation mechanism was anisotropic. This was readily apparent when the output of the laser-pumped dye laser was strongly polarized in spite of the fact that the resonator and the dye solution were otherwise isotropic. ${ }^{1}$ The anisotropic pumping scheme produced a gain that was no longer uniform with respect to the polarization of the laser field.

Other early studies of anisotropic pumping concerned the fluorescent emission from atoms and molecules. The fluorescent emission of an atom or molecule excited by linearly polarized light has been found to possess a definite polarization characteristic. ${ }^{2}$ From electromagnetic theory it is known that the elementary radiators, that is, oscillating electric or magnetic dipoles, rotors, and quadrupoles produce polarized electromagnetic waves. The emission process from a single atom or molecule is usually modeled by one or more of these radiators, but whether or not the overall fluorescent emission from an ensemble of atoms or molecules is polarized depends on the orientation of the radiators in the ensemble. A random orientation produces an overall unpolarized emission, whereas the existence of some preferential direction favors a polarized emission. This preferential direction may be provided by the linear polarization of the pump source. When both the absorption and emission processes are modeled by elementary oscillators, nearly all known cases of polarized fluorescence can be explained. ${ }^{3}$

The polarization characteristics of optically pumped media are usually described in terms of the degree of polarization, defined for excitation by linearly polarized radiation. The value of $P$ is found from the equation

$$
P=\left(I_{\|}-I_{1}\right) /\left(I_{\|}+I_{1}\right),
$$

where $I_{\|}$is the intensity of emission parallel to the exciting field and $I_{\perp}$ is the intensity of emission perpendicular to the exciting field. For all cases involving linear oscillators it has been shown that $P$ is less than or equal to one half. ${ }^{3}$ The particular value of $P$ depends on which elementary oscillator can be used to describe the absorption and emission processes.

The interesting absorption and emission bands of organic dye molecules occur primarily in the visible region of the spectrum. This is believed to be due to a molecular structure that contains a chain of conjugated double bonds. ${ }^{4,5}$ It has been shown that for most purposes the interaction of radiation with a chain of conjugated double bonds can be described by a partially anisotropic linear oscillator rigidly connected to the chain. ${ }^{6}$ The direction of the linear oscillator has been shown to be parallel with the axis of the chain, ${ }^{7}$ which in turn is the unique axis of the molecular coordinate system. The interaction between the pump electric field and the dye molecule depends only on the angle between the exciting field and the unique axis of the dye molecule. This model provides a means for including the factors that infiuence $P$, specifically the angle between the oscillators used for absorption and emission. This angle is a function of the structure of the molecule, the wavelength of the pump, the concentration of dye molecules, the solvent, the temperature, and any impurities that are present. ${ }^{8}$

The first successful explanation of the polarization effects in dye lasers was made by Sevchenko and coworkers. ${ }^{9}$ They modeled the absorption and emission processes by electric dipole transitions and assumed that the dipoles were parallel and fixed with respect to the molecules. The molecules themselves were uniformly distributed and rigidly fixed so that there could be no molecular motion from excitation till emission. The effect of the linearly polarized pump 


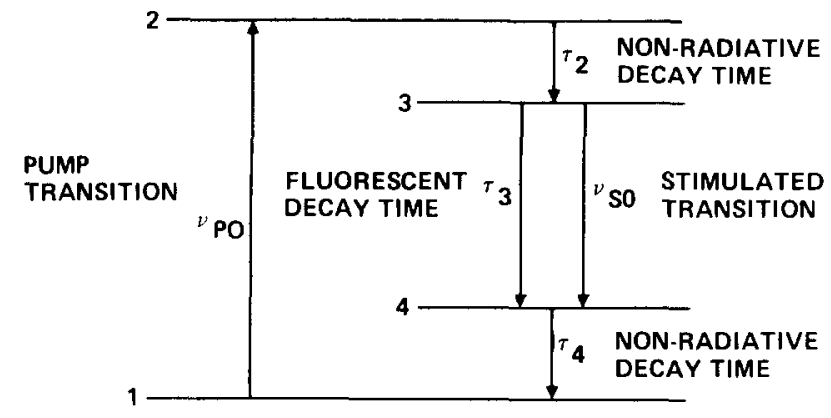

FIG. 1. Energy level model used in laser analysis.

was to preferentially excite those molecules that were oriented in a direction close to that of the pump, and the result was a nonuniform distribution of excited molecules and hence an anisotropic gain. The gain was found to possess a maximum along the direction of the pump field polarization. The main points of the current dye-laser polarization theory were recently reviewed by Lempicki, ${ }^{10}$ and he emphasized that none of the previous treatments had included saturation effects and that rotational diffusion had only been included at threshold. " "The primary objective of this study is to develop a laser model in which saturation effects are fully included. Emphasis here is on lasers having unidirectional molecular distributions, and isotropic distributions are treated in a companion paper. ${ }^{12}$ The effects of rotational diffusion are discussed in a related study. ${ }^{13}$

The basic semiclassical formalism for treating anisotropically pumped laser amplifiers is developed in Sec. II, and explicit formulas are obtained for the population differences of the pump and signal transitions. The field equations are derived in Sec. III, and the limit of line center tuning with no saturation is considered in detail. Analytical solutions for the special case of a unidirectional distribution are presented in Sec. IV. Whenever possible, the results are described in terms of intuitive physical models of the dipole-field interactions. Under a broad range of conditions the polarization of a propagating signal tends to rotate with distance toward the orientation of the molecules, while the pump tends to rotate toward the perpendicular direction.

\section{SEMICLASSICAL THEORY}

In this section a theory is developed for the gain anisotropies in a laser amplifier resulting from the polarization of the pump source. The starting point for this treatment is the familiar density matrix equations, but they are extended here to include the pumping field as a coherent interaction rather than as an incoherent term that is added phenomenologically. In this way, the vector properties of the pump and signal fields are automatically included.

\section{A. Density matrix model}

The energy-level model that is used in the development of the amplifier theory is the four-level system shown in Fig. 1. It is assumed that the pump absorption takes place only between levels one and two while stimulated emission takes place only betwen levels three and four. The coupling be- tween the absorption levels and the emission levels is taken to be nonradiative relaxation with a relaxation time $\tau_{2}$ between levels two and three, and $\tau_{4}$ between levels four and one. Spontaneous emission is included between levels three and four with a fluorescent decay time $\tau_{3}$. For the case of a dye laser, levels one and four would be members of a single vibrational manifold as would levels two and three.

The procedure for setting up the density matrix equations is well known, ${ }^{14}$ and the results for this energy level model may be written

$$
\begin{aligned}
\frac{\partial \rho_{11}}{\partial t}=- & \frac{i}{\hbar}\left(\rho_{12} \boldsymbol{\mu}_{21}-\rho_{21} \boldsymbol{\mu}_{12}\right) \cdot \mathbf{E}_{p}+\frac{\rho_{44}}{\tau_{4}}, \\
\frac{\partial \rho_{22}}{\partial t} & =\frac{i}{\hbar}\left(\rho_{12} \boldsymbol{\mu}_{21}-\rho_{21} \boldsymbol{\mu}_{12}\right) \cdot \mathbf{E}_{p}-\frac{\rho_{22}}{\tau_{2}}, \\
\frac{\partial \rho_{33}}{\partial t} & =-\frac{i}{\hbar}\left(\rho_{34} \boldsymbol{\mu}_{43}-\rho_{43} \boldsymbol{\mu}_{34}\right) \cdot \mathbf{E}_{s}+\frac{\rho_{22}}{\tau_{2}}-\frac{\rho_{33}}{\tau_{3}}, \\
\frac{\partial \rho_{44}}{\partial t} & =\frac{i}{\hbar}\left(\rho_{34} \boldsymbol{\mu}_{43}-\rho_{43} \boldsymbol{\mu}_{34}\right) \cdot \mathbf{E}_{s}+\frac{\rho_{33}}{\tau_{3}}-\frac{\rho_{44}}{\tau_{4}}, \\
\frac{\partial \rho_{21}}{\partial t} & =-i \omega_{p 0} \rho_{21}+\frac{i}{\hbar}\left(\rho_{11}-\rho_{22}\right) \boldsymbol{\mu}_{12} \cdot \mathbf{E}_{p}-\frac{\rho_{21}}{T_{p}}, \\
\frac{\partial \rho_{34}}{\partial t} & =-i \omega_{50} \rho_{34}-\frac{i}{\hbar}\left(\rho_{33}-\rho_{44}\right) \boldsymbol{\mu}_{34} \cdot \mathbf{E}_{s}-\frac{\rho_{34}}{T_{s}}, \\
\rho_{12} & =\rho_{21}^{*}, \\
\rho_{43} & =\rho_{34}^{*},
\end{aligned}
$$

where $\mathbf{E}_{p}$ and $\mathbf{E}_{s}$ are respectively the pump and signal electric fields, $\omega_{p 0}$ and $\omega_{s 0}$ are respectively the center frequencies of the pump and signal transitions, the various $\mu_{j i}$ are the matrix elements of the dipole moment operator, and $T_{p}$ and $T_{s}$ are the coherence times of the off-diagonal matrix elements. Multiplying Eq. (6) by $\mu_{12}$ and Eq. (7) by $\mu_{43}$ and moving these terms inside of the derivatives since they are time independent in the Schrödinger representation yields

$$
\begin{aligned}
& \frac{\partial \boldsymbol{\eta}_{21}}{\partial t}=-i \omega_{p 0} \boldsymbol{\eta}_{21}+\frac{i}{\hbar} D_{p}\left|\mu_{p}\right|^{2}\left(\mathbf{E}_{p} \cdot \hat{e}_{p}\right) \hat{e}_{p}-\frac{\boldsymbol{\eta}_{21}}{T_{p}}, \\
& \frac{\partial \boldsymbol{\eta}_{34}}{\partial t}=-i \omega_{s 0} \boldsymbol{\eta}_{34}-\frac{i}{\hbar} D_{s}\left|\mu_{s}\right|^{2}\left(\mathbf{E}_{s} \cdot \hat{e}_{s}\right) \hat{e}_{s}-\frac{\boldsymbol{\eta}_{34}}{T_{s}}
\end{aligned}
$$

where

$$
\begin{aligned}
& \eta_{21}=\rho_{21} \mu_{12}=\rho_{21} \mu_{12} \hat{e}_{p}, \\
& \eta_{34}=\rho_{34} \mu_{43}=\rho_{34} \mu_{43} \hat{e}_{s}, \\
& \left|\mu_{p}\right|^{2}=\mu_{21} \mu_{21}^{*}=\mu_{21} \mu_{12}, \\
& \left|\mu_{s}\right|^{2}=\mu_{34} \mu_{34}^{*}=\mu_{34} \mu_{43}, \\
& D_{p}=\rho_{11}-\rho_{22}, \\
& D_{s}=\rho_{33}-\rho_{44},
\end{aligned}
$$

and $\hat{e}_{s}$ and $\hat{e}_{p}$ are unit vectors in the directions of $\mu_{43}$ and $\mu_{12}$ respectively.

The use of the density matrix provides a means for obtaining the average induced dipole moment for an ensemble of molecules. Consider a molecule whose transition moment is oriented within the solid angle $d \Omega$ about the direction giv- 


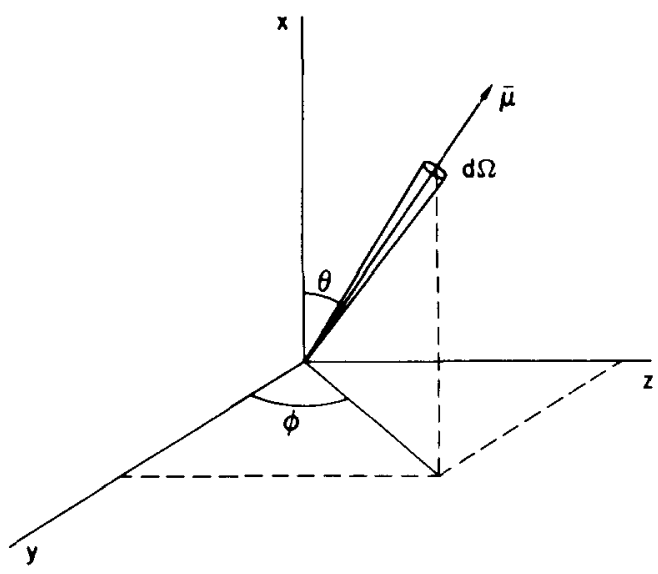

FIG. 2. Coordinate geometry used to characterize the molecular dipole moments.

en by the spherical coordinates $(\theta, \phi)$ as shown in Fig. 2. If all molecules found in this solid angle are considered as a class, then the average induced dipole moment due to this class of dipoles is found by taking the ensemble average of the expectation value of the dipole moment operator for each molecule in the class. ${ }^{15}$ Then Eqs. (2)-(5) and (8)-(11) become a complete set of equations for the matrix elements of $\rho_{p}$ and $\rho_{s}$ for one class of dipoles.

The average value of the dipole moment induced by the pump and stimulating fields for one class of dipoles is given by $^{16}$

$$
\begin{aligned}
& \left\langle\boldsymbol{\mu}_{p}\right\rangle_{\mathrm{av}}=\rho_{12} \boldsymbol{\mu}_{21}+\rho_{21} \boldsymbol{\mu}_{12}, \\
& \left\langle\boldsymbol{\mu}_{s}\right\rangle_{\mathrm{av}}=\rho_{34} \boldsymbol{\mu}_{43}+\rho_{43} \boldsymbol{\mu}_{34},
\end{aligned}
$$

where \langle\rangle$_{\mathrm{av}}$ means the ensemble average of the expectation value. The actual induced moment is the sum of the expressions in Eqs. (18). However, the dipole induced by the pump field and the dipole induced by the stimulating field are oscillating at different frequencies, and it is assumed that the frequency difference is large enough for the rotating wave approximation to apply. This approximation means that only Eq. (18a) will be used as the source term for the pump field and only Eq. (18b) will be used as the source term for the stimulating field. In the case of dye molecules, this is a good approximation due to the large Stokes shift between the absorbing and emitting frequencies (e.g., Rhodamine $6 \mathrm{G}$ has an absorption peak at $532 \mathrm{~nm}$ and an emission peak at 590 $\mathrm{nm}$ so $v_{p}-v_{s}=5.54 \times 10^{13} \mathrm{~Hz}$ ). ${ }^{17}$

The total polarization $\mathbf{P}$ resulting from the induced dipole moments is found by integrating the macroscopic polarization due to one class of dipoles over all classes. This can be expressed as

$$
\begin{aligned}
& \mathbf{P}_{p}=\int_{\Omega} n(\theta, \phi)\left\langle\mu_{p}\right\rangle_{\mathrm{av}} d \Omega, \\
& \mathbf{P}_{s}=\int_{\Omega} n(\theta, \phi)\left\langle\mu_{s}\right\rangle_{\mathrm{av}} d \Omega,
\end{aligned}
$$

where $n(\theta, \phi) d \Omega$ is the number of molecules per unit volume oriented within the solid angle $d \Omega$ about the $(\theta, \phi)$ direction.

\section{B. Population differences}

A solution to the set of Eqs. (2)-(5) and Eqs. (8)-(11) can be obtained assuming the pump and stimulating fields have a plane wave dependence and the propagation vectors $k_{p}$ and $\mathbf{k}_{s}$ are colinear. Referring to the coordinate system in Fig. 3, $\mathbf{E}_{p}$ and $\mathbf{E}_{s}$ can be written

$$
\begin{aligned}
& \mathbf{E}_{p}(z, t)=\frac{1}{2} \mathscr{E}_{p}(z, t) \exp \left[i\left(k_{p} z-\omega_{p} t\right)\right]+\text { c.c., } \\
& \mathbf{E}_{s}(z, t)=\frac{1}{2} \mathscr{E}_{s}(z, t) \exp \left[i\left(k_{s} z-\omega_{s} t\right)\right]+\text { c.c., }
\end{aligned}
$$

where $\mathscr{E}_{p}(z, t)$ and $\mathscr{E}_{s}(z, t)$ are slowly varying complex vector functions of $z$ and $t$. The plane wave dependence in the $\eta_{21}$ and $\eta_{34}$ terms from Eqs. (10) and (11) can be factored out as

$$
\begin{aligned}
& \eta_{21}(\theta, \phi, z, t)=\eta_{p}(\theta, \phi, z, t) \exp \left[i\left(k_{p} z-\omega_{p} t\right)\right] \hat{e}_{p}, \\
& \eta_{34}(\theta, \phi, z, t)=\eta_{s}(\theta, \phi, z, t) \exp \left[i\left(k_{s} z-\omega_{s} t\right)\right] \hat{e}_{s},
\end{aligned}
$$

where $\eta_{p}$ and $\eta_{s}$ are complex functions that vary slowly in $z$ and $t$. Equations (20) and (21) along with the rotating wave approximation in Eqs. (2)-(5) and (8)-(11) yields

$$
\begin{aligned}
\frac{\partial \rho_{11}}{\partial t}=- & \frac{1}{\hbar} \operatorname{Im}\left(\boldsymbol{\eta}_{p} \cdot \mathscr{E}_{p}^{*}\right)+\frac{\rho_{44}}{\tau_{4}}, \\
\frac{\partial \rho_{22}}{\partial t} & =\frac{1}{\hbar} \operatorname{Im}\left(\boldsymbol{\eta}_{p} \cdot \mathscr{E}_{p}^{*}\right)-\frac{\rho_{22}}{\tau_{2}}, \\
\frac{\partial \rho_{33}}{\partial t} & =\frac{1}{\hbar} \operatorname{Im}\left(\boldsymbol{\eta}_{s} \cdot \mathscr{E}_{s}^{*}\right)+\frac{\rho_{22}}{\tau_{2}}-\frac{\rho_{33}}{\tau_{3}}, \\
\frac{\partial \rho_{44}}{\partial t} & =-\frac{1}{\hbar} \operatorname{Im}\left(\boldsymbol{\eta}_{s} \cdot \mathscr{E}_{s}^{*}\right)+\frac{\rho_{33}}{\tau_{3}}-\frac{\rho_{44}}{\tau_{4}}, \\
\frac{\partial \eta_{p}}{\partial t} & =i\left(\omega_{p}-\omega_{p 0}\right) \eta_{p}+\frac{i}{2 \hbar} D_{p}\left|\mu_{p}\right|^{2}\left(\mathscr{E}_{p} \cdot \hat{e}_{p}\right)-\frac{\eta_{p}}{T_{p}}, \\
\frac{\partial \eta_{s}}{\partial t} & =i\left(\omega_{s}-\omega_{s 0}\right) \eta_{s}-\frac{i}{2 \hbar} D_{s}\left|\mu_{s}\right|^{2}\left(\mathscr{E}_{s} \cdot \hat{e}_{s}\right)-\frac{\eta_{s}}{T_{s}},
\end{aligned}
$$

where $\boldsymbol{\eta}_{p}=\eta_{p} \hat{e}_{p}$ and $\boldsymbol{\eta}_{s}=\eta_{s} \hat{e}_{s}$.

The response of the laser medium to the pump and signal fields is now described completely by the six equations,

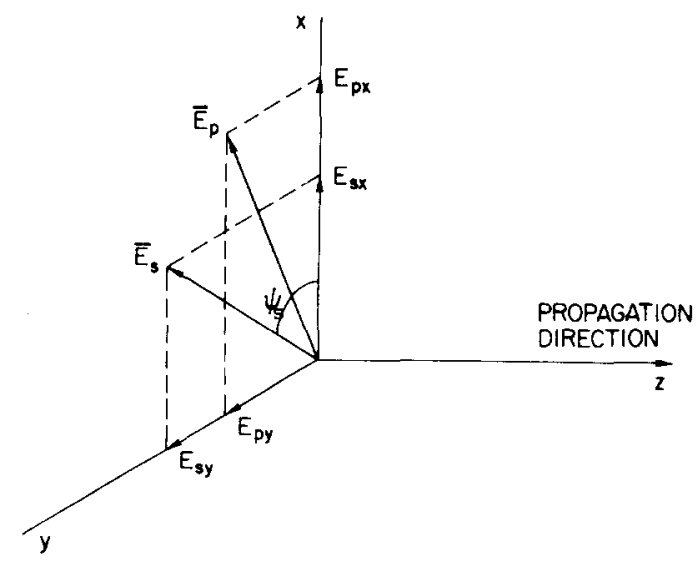

FIG. 3. Geometry of pump and stimulating electromagnetic fields. 
Eqs. (22)-(26). When the fields are known, these equations can be solved for the population differences $D_{p}$ and $D_{s}$. However, several simplifications are often possible. First of all, Eqs. (26a) and (26b) can be solved analytically if it is assumed that the coherence times are much shorter than any other times of interest. The derivatives are set equal to zero and the solutions become

$\eta_{p}=-\left|\mu_{p}\right|^{2} D_{p} \mathscr{L}\left(v_{p}\right)\left(\mathscr{C}_{p} \cdot \hat{e}_{p}\right)\left[\left(\omega_{p}-\omega_{p 0}\right) T_{p}-i\right] / 4 \hbar$,

$\eta_{s}=\left|\mu_{s}\right|^{2} D_{s} \mathscr{L}\left(\nu_{s}\right)\left(\mathscr{E}_{s} \cdot \hat{e}_{s}\right)\left[\left(\omega_{s}-\omega_{s 0}\right) T_{p}-i\right] / 4 \hbar$,

where $\mathscr{L}\left(v_{p}\right)$ and $\mathscr{L}\left(v_{s}\right)$ are the normalized Lorentzian lineshape functions given by

$$
\begin{aligned}
& \mathscr{L}\left(v_{p}\right)=\frac{2 / \pi \Delta v_{p}}{1+\left[2\left(v_{p}-v_{p 0}\right) / \Delta v_{p}\right]^{2}}, \\
& \mathscr{L}\left(v_{s}\right)=\frac{2 / \pi \Delta v_{s}}{1+\left[2\left(v_{s}-v_{\mathrm{s} 0}\right) / \Delta v_{s}\right]^{2}},
\end{aligned}
$$

and $\Delta v_{p}$ and $\Delta v_{s}$ are the full widths at half maximum and are related to the coherence times by $\Delta v_{p}=\left(\pi T_{p}\right)^{-1}$ and $\Delta v_{s}$ $=\left(\pi T_{s}\right)^{-1}$. When these results are substituted into Eqs. (22) $-(25)$, the laser problem is reduced to four coupled equations.

If the pump field is taken to be $\mathrm{cw}$ or quasi-cw (i.e., the duration of the pump pulse is much longer than the effective lifetime of the excited states), Eqs. (23)-(25) can be solved analytically for the population differences $D_{p}$ and $D_{s}$. The solution is obtained by setting the derivatives equal to zero and inserting the results from Eq. (27). After some algebraic manipulation one obtains

$D_{p}=\frac{1+\tau_{3} \beta_{s}}{1+\left(2 \tau_{2}+\tau_{3}+\tau_{4}\right) \beta_{p}+\tau_{3} \beta_{s}+2 \tau_{3}\left(\tau_{2}+\tau_{4}\right) \beta_{p} \beta_{s}}$,

$D_{s}=\frac{\left(\tau_{3}-\tau_{4}\right) \beta_{p}}{1+\left(2 \tau_{2}+\tau_{3}+\tau_{4}\right) \beta_{p}+\tau_{3} \beta_{s}+2 \tau_{3}\left(\tau_{2}+\tau_{4}\right) \beta_{p} \beta_{s}}$,

where

$$
\begin{gathered}
\beta_{p}=\left|\mu_{p}\right|^{2}\left|\mathscr{E}_{p} \cdot \hat{e}_{p}\right|^{2} \mathscr{L}\left(v_{p}\right) \mid 4 \hbar^{2}, \\
\beta_{s}=\left|\mu_{s}\right|^{2}\left|\mathscr{E}_{s} \cdot \hat{e}_{s}\right|^{2} \mathscr{L}\left(v_{s}\right) \mid 4 \hbar^{2},
\end{gathered}
$$

The expressions in Eqs. (29) can be simplified further by requiring that the absorption and emission dipoles be parallel, that is $\hat{e}_{p}=\hat{e}_{s}$. This is reasonable in a dye solution as long as the pump source is not in the ultraviolet. With ultraviolet pumping the dye molecules may be excited to the second excited singlet level. There is a nonradiative relaxation from that level to the first excited singlet state after which a radiative transition can occur. Since the two excited singlet levels represent different electronic states, the transition moments are not necessarily parallel. ${ }^{18}$ On the other hand, when the pump source is in the visible, the molecules are excited to an upper vibrational level of the same electronic state and consequently the absorption and emission dipoles must be parallel.
To be specific, we also require that the relaxation time for the nonradiative decay from levels two and four shown in Fig. 1 is much less than the effective lifetime of level three. In a dye, the vibrational relaxation times are on the order of picoseconds while the effective lifetime of the emitting level ranges from tens of picoseconds to a few nanoseconds depending on the magnitude of the stimulating field. Thus, in the expressions for the population differences in Eqs. (29) $\tau_{2}$ and $\tau_{4}$ are set equal to zero.

The functions $\mathscr{E}_{p}(z, t)$ and $\mathscr{E}_{s}(z, t)$ can be expanded as

$$
\begin{aligned}
\mathscr{E}_{p}(z, t)= & E_{p x}(z, t) \exp \left[i \delta_{p x}(z, t)\right] \hat{e}_{x} \\
& +E_{p y}(z, t) \exp \left[i \delta_{p y}(z, t)\right] \hat{e}_{y}, \\
\mathscr{E}_{s}(z, t)= & E_{s x}(z, t) \exp \left[i \delta_{s x}(z, t)\right] \hat{e}_{x} \\
& +E_{s y}(z, t) \exp \left[i \delta_{s y}(z, t)\right] \hat{e}_{y},
\end{aligned}
$$

where $E_{\rho x}, E_{p y}, E_{x x}$, and $E_{s y}$ are the real, orthogonal amplitude components of $\mathbf{E}_{p}$ and $\mathbf{E}_{s}$, and $\hat{e}_{x}$ and $\hat{\boldsymbol{e}}_{y}$ are the unit vectors in the $x$ and $y$ directions respectively. With the above simplifications, the population differences become

$$
\begin{aligned}
& D_{p}(\theta, \phi, z, t)=\frac{1+f_{s}(\theta, \phi, z, t)}{1+f_{p}(\theta, \phi, z, t)+f_{s}(\theta, \phi, z, t)}, \\
& D_{s}(\theta, \phi, z, t)=\frac{f_{p}(\theta, \phi, z, t)}{1+f_{\rho}(\theta, \phi, z, t)+f_{s}(\theta, \phi, z, t)},
\end{aligned}
$$

where

$$
\begin{aligned}
f_{p}= & \Phi_{p x}^{2} \cos ^{2} \theta+2 \Phi_{p x} \Phi_{p y} \cos \delta_{p} \cos \theta \sin \theta \cos \phi \\
& +\Phi_{p y}^{2} \sin ^{2} \theta \cos ^{2} \phi, \\
f_{s}= & \Phi_{s x}^{2} \cos ^{2} \theta+2 \Phi_{s x} \Phi_{s y} \cos \delta_{s} \cos \theta \sin \theta \cos \phi \\
& +\Phi_{s y}^{2} \sin ^{2} \theta \cos ^{2} \phi .
\end{aligned}
$$

The terms $\Phi_{p x}, \Phi_{p y}, \Phi_{s x}$, and $\Phi_{s y}$ are dimensionless field amplitudes given by

$$
\begin{aligned}
& \Phi_{p x}=\left|\mu_{p}\right|\left[\tau_{3} \mathscr{L}\left(v_{p}\right)\right]^{1 / 2} E_{p x} / 2 \hbar, \\
& \Phi_{p y}=\left|\mu_{p}\right|\left[\tau_{3} \mathscr{L}\left(v_{p}\right)\right]^{1 / 2} E_{p y} / 2 \hbar, \\
& \Phi_{s x}=\left|\mu_{s}\right|\left[\tau_{3} \mathscr{L}\left(v_{s}\right)\right]^{1 / 2} E_{s x} / 2 \hbar, \\
& \Phi_{s y}=\left|\mu_{s}\right|\left[\tau_{3} \mathscr{L}\left(v_{s}\right)\right]^{1 / 2} E_{s y} / 2 \hbar .
\end{aligned}
$$

These dimensionless field amplitudes may also be related to the intensity of the $x$ and $y$ components of the pump and stimulating fields $\left(I_{p x}, I_{p y}, I_{s x}\right.$, and $\left.I_{s y}\right)$ by

$$
\begin{aligned}
& \Phi_{p x}^{2}=\tau_{3} \sigma_{p}\left(v_{p}\right) I_{p x} / h v_{p}, \\
& \Phi_{p y}^{2}=\tau_{3} \sigma_{p}\left(v_{p}\right) I_{p y} / h v_{p}, \\
& \Phi_{s x}^{2}=\tau_{3} \sigma_{s}\left(v_{s}\right) I_{s x} / h v_{s}, \\
& \Phi_{s y}^{2}=\tau_{s} \sigma_{s}\left(v_{s}\right) I_{s y} / h v_{s},
\end{aligned}
$$

where $\sigma_{p}\left(v_{p}\right)$ is the absorption cross section given by

$$
\sigma_{p}\left(v_{p}\right)=h v_{p} n_{0}\left|\mu_{p}\right|^{2} \mathscr{L}\left(v_{p}\right) / 2 \epsilon c \hbar^{2}
$$

and $\sigma_{s}\left(v_{s}\right)$ is the stimulated emission cross section given by

$$
\sigma_{s}\left(\nu_{s}\right)=h v_{s} n_{0}\left|\mu_{s}\right|^{2} \mathscr{L}\left(v_{s}\right) / 2 \epsilon c \hbar^{2} .
$$

As an example, rhodamine $6 \mathrm{G}$ has a cross section for absorption and stimulated emission approximately equal to $2 \times 10^{-16} \mathrm{~cm}^{2}$ and a fluorescent decay time of approximately $5 \times 10^{-9} \mathrm{sec}^{19}$ Therefore, a pump intensity of $125 \mathrm{~kW} / \mathrm{cm}^{2}$ at 
$532 \mathrm{~nm}$ and a signal intensity of $105 \mathrm{~kW} / \mathrm{cm}^{2}$ at $590 \mathrm{~nm}$ would correspond to a value of unity for the dimensionless field amplitudes.

\section{ELECTROMAGNETIC FIELD EQUATIONS A. Arbitrary frequency}

The differential equations that the pump and stimulating fields satisfy are derived from the vector wave equation by separating $\mathbf{E}_{p}$ and $\mathbf{E}_{s}$ into orthogonal components and using the macroscopic polarizations $\mathbf{P}_{p}$ and $\mathbf{P}_{s}$ from Eqs. (19) as the source terms. The initial equations are

$$
\begin{aligned}
& \frac{\partial^{2} \mathbf{E}_{p}}{\partial z^{2}}-\mu \epsilon \frac{\partial^{2} \mathbf{E}_{p}}{\partial t^{2}}-\mu \sigma \frac{\partial \mathbf{E}_{p}}{\partial t}=\mu \frac{\partial^{2} \mathbf{P}_{p}}{\partial t^{2}}, \\
& \frac{\partial^{2} \mathbf{E}_{s}}{\partial z^{2}}-\mu \epsilon \frac{\partial^{2} \mathbf{E}_{s}}{\partial t^{2}}-\mu \sigma \frac{\partial \mathbf{E}_{s}}{\partial t}=\mu \frac{\partial^{2} \mathbf{P}_{s}}{\partial t^{2}} .
\end{aligned}
$$

Using Eqs. (18)-(21) along with Eqs. (31), the first of these wave equations may be decomposed in to the set of amplitude and phase equations

$$
\begin{aligned}
\frac{\partial E_{p x}}{\partial z} & +\frac{n_{0}}{c} \frac{\partial E_{p x}}{\partial t}+\frac{\gamma_{p}}{2} E_{p x} \\
& =-\frac{\alpha_{x}}{2} E_{p x}-\frac{\alpha_{x y}}{2} E_{p y}\left(\cos \delta_{p}+K_{p} \sin \delta_{p}\right) \\
\frac{\partial E_{p y}}{\partial z}+ & \frac{n_{0}}{c} \frac{\partial E_{p y}}{\partial t}+\frac{\gamma_{p}}{2} E_{p y} \\
= & -\frac{\alpha_{y}}{2} E_{p y}-\frac{\alpha_{x y}}{2} E_{p x}\left(\cos \delta_{p}-K_{p} \sin \delta_{p}\right) \\
\frac{\partial \delta_{p}}{\partial z}+ & \frac{n_{0}}{c} \frac{\partial \delta_{p}}{\partial t} \\
= & \frac{\alpha_{x y}}{2}\left(K_{p} \frac{E_{p x}^{2}-E_{p y}^{2}}{E_{p x} E_{p y}} \cos \delta_{p}+\frac{E_{p x}^{2}+E_{p y}^{2}}{E_{p x} E_{p y}} \sin \delta_{p}\right) \\
& -\frac{K_{p}\left(\alpha_{x}-\alpha_{y}\right)}{2},
\end{aligned}
$$

where the higher-order space and time derivatives of slowly varying quantities have been neglected and the rotating wave approximation has been employed. The parameters $\alpha_{x}, \alpha_{x y}$, and $\alpha_{y}$ are the absorption coefficients which are expressed as

$$
\begin{aligned}
\alpha_{x}(z, t)= & \sigma_{p}\left(v_{p}\right) \int_{\Omega} n(\theta, \phi) D_{p}(\theta, \phi, z, t) \\
& \times \cos ^{2} \theta d \Omega, \\
\alpha_{x y}(z, t)= & \sigma_{p}\left(v_{p}\right) \int_{\Omega} n(\theta, \phi) D_{p}(\theta, \phi, z, t) \\
& \times \cos \theta \sin \theta \cos \phi d \Omega, \\
\alpha_{y}(z, t)= & \sigma_{p}\left(v_{p}\right) \int_{\Omega} n(\theta, \phi) D_{p}(\theta, \phi, z, t) \\
& \times \sin ^{2} \theta \cos ^{2} \phi d \Omega .
\end{aligned}
$$

The phase difference $\delta_{p}$ and the off-line-center parameter $K_{p}$ in Eqs. (39) are given by

$$
\begin{aligned}
& \delta_{p}(z, t)=\delta_{p x}(z, t)-\delta_{p y}(z, t), \\
& K_{p}=2\left(v_{p}-v_{p 0}\right) / \Delta v_{p} .
\end{aligned}
$$

and $\gamma_{p}$ represents any additional losses other than by resonant absorption. The propagation constants are given by $k_{p}^{2}$ $=\mu \epsilon \omega_{p}^{2}$ and $k_{s}^{2}=\mu \epsilon \omega_{s}^{2}$, and the phase velocity is $c / n_{0}=(\mu \epsilon)^{-1 / 2}$.

In a similar manner, the equations for the stimulating field components and phase difference can be obtained. These equations are

$$
\begin{aligned}
\frac{\partial E_{s x}}{\partial z}+ & \frac{n_{0}}{c} \frac{\partial E_{s x}}{\partial t}+\frac{\gamma_{s}}{2} E_{s x} \\
& =\frac{g_{x}}{2} E_{s x}+\frac{g_{x y}}{2} E_{s y}\left(\cos \delta_{s}+K_{s} \sin \delta_{s}\right) \\
\frac{\partial E_{s y}}{\partial z}+ & \frac{n_{0}}{c} \frac{\partial E_{s y}}{\partial t}+\frac{\gamma_{s}}{2} E_{s y} \\
= & \frac{g_{y}}{2} E_{s y}+\frac{g_{x y}}{2} E_{s x}\left(\cos \delta_{s}-K_{s} \sin \delta_{s}\right) \\
\frac{\partial \delta_{s}}{\partial z}+ & \frac{n_{0}}{c} \frac{\partial \delta_{s}}{\partial t} \\
= & -\frac{g_{x y}}{2}\left(K_{s} \frac{E_{s x}^{2}-E_{s y}^{2}}{E_{s x} E_{s y}} \cos \delta_{s}+\frac{E_{s x}^{2}+E_{s y}^{2}}{E_{s x} E_{s y}} \sin \delta_{s}\right) \\
& \quad+\frac{K_{s}\left(g_{x}-g_{y}\right)}{2},
\end{aligned}
$$

where $g_{x}, g_{x y}$, and $g_{y}$ are the gain coefficients expressed as

$$
\begin{aligned}
g_{x}(z, t)= & \sigma_{s}\left(v_{s}\right) \int_{\Omega} n(\theta, \phi) D_{s}(\theta, \phi, z, t) \\
& \times \cos ^{2} \theta d \Omega, \\
g_{x y}(z, t)= & \sigma_{s}\left(v_{s}\right) \int_{\Omega} n(\theta, \phi) D_{s}(\theta, \phi, z, t) \\
& \times \cos \theta \sin \theta \cos \phi d \Omega, \\
g_{y}(z, t)= & \sigma_{s}\left(v_{s}\right) \int_{\Omega} n(\theta, \phi) D_{s}(\theta, \phi, z, t) \\
& \times \sin ^{2} \theta \cos ^{2} \phi d \Omega .
\end{aligned}
$$

The phase difference $\delta_{s}$ and the off-line-center parameter $K_{s}$ in Eqs. (43) are given by

$$
\begin{aligned}
& \delta_{s}(z, t)=\delta_{s x}(z, t)-\delta_{s y}(z, t), \\
& K_{s}=2\left(v_{s}-v_{s_{0}}\right) / \Delta v_{s},
\end{aligned}
$$

and $\gamma_{s}$ represents any losses incurred by the stimulating field.

Equations (39) and (44) are a set of six partial differential equations in the six unknowns $E_{p x}, E_{p y}, E_{s x}, E_{s y}, \delta_{p}$, and $\delta_{s}$. These equations become a complete set when it is recalled that the population differences are related to the fields by means of the density matrix equations of the previous section. With quasi-cw operation the density matrix equations have been reduced to Eqs. (32). The initial conditions at $z=0$ are known since they are the values of the incident pump and stimulating fields. These equations can be solved in general by numerical methods. However, certain special cases allow a substantial simplification of the equations and still lead to physically useful solutions. 


\section{B. Line center tuning}

When the pump and signal fields are tuned to the linecenter frequency of their respective transitions, the parameters $K_{p}$ and $K_{s}$ from Eqs. (42) and (46) vanish. Then the phase difference equations become, for a cw laser,

$$
\begin{aligned}
& \frac{d \delta_{p}}{d z}=\frac{1}{2} \alpha_{x y} \frac{E_{p x}^{2}+E_{p y}^{2}}{E_{p x} E_{p y}} \sin \delta_{p}, \\
& \frac{d \delta_{s}}{d z}=-\frac{1}{2} g_{x y} \frac{E_{s x}^{2}+E_{s y}^{2}}{E_{s x} E_{s y}} \sin \delta_{s} .
\end{aligned}
$$

When the pump and signal fields are linearly polarized at the input plane $(z=0), \delta_{p}(0)$ and $\delta_{s}(0)$ are zero and so the derivatives in Eqs. (47) are also zero at $z=0$. This means that the phase differences are zero for all $z$ and thus the pump and signal fields remain linearly polarized as they propagate in the laser medium. This is important for practical applications since many laser pump and signal sources are linearly polarized.

It is convenient now to transform Eqs. (39a), (39b), (43a), and (43b) to a new set governing the total field amplitude and polarization orientation for both the pump and the signal. To achieve this transformation for the signal equations, one can first multiply Eq. (43a) by $2 E_{s x}$ and Eq. (43b) by $2 E_{s y}$ and then add to obtain

$$
\begin{aligned}
& \frac{\partial\left(E_{s x}^{2}+E_{s y}^{2}\right)}{\partial z}+\frac{n_{0}}{c} \frac{\partial\left(E_{s x}^{2}+E_{s y}^{2}\right)}{\partial t}+\gamma_{s}\left(E_{s x}^{2}+E_{s y}^{2}\right) \\
& =g_{x} E_{s x}^{2}+2 g_{x y} E_{s x} E_{s y}+g_{y} E_{s y}^{2} .
\end{aligned}
$$

But $E_{s x}$ and $E_{s y}$ can be written in terms of the total field amplitude $E_{s}$ and the polarization angle $\psi_{s}$ with respect to the $x$ axis as

$$
\begin{aligned}
& E_{s x}=E_{s} \cos \psi_{s}, \\
& E_{s y}=E_{s} \sin \psi_{s} .
\end{aligned}
$$

Thus Eq. (48) becomes

$$
\begin{aligned}
\frac{\partial E_{s}^{2}}{\partial z} & +\frac{n_{0}}{c} \frac{\partial E_{s}^{2}}{\partial t}+\gamma_{s} E_{s}^{2} \\
& =\left(g_{x} \cos ^{2} \psi_{s}+2 g_{x y} \cos \psi_{s} \sin \psi_{s}+g_{y} \sin ^{2} \psi_{s}\right) E_{s}^{2}
\end{aligned}
$$

or, with the expansion of the derivatives,

$$
\begin{aligned}
\frac{\partial E_{s}}{\partial z} & +\frac{n_{0}}{c} \frac{\partial E_{s}}{\partial t}+\frac{\gamma_{s}}{2} E_{s} \\
& =\frac{1}{2}\left(g_{x} \cos ^{2} \psi_{s}+2 g_{x y} \cos \psi_{s} \sin \psi_{s}+g_{y} \sin ^{2} \psi_{s}\right) E_{s} .
\end{aligned}
$$

A similar equation can be derived describing the variations of the polarization angle $\psi_{s}$. If Eq. (49a) is substituted directly into Eq. (43a) and the result is divided by $\cos \psi_{s}$, one obtains

$$
\begin{aligned}
\frac{\partial E_{s}}{\partial z} & +\frac{n_{0}}{c} \frac{\partial E_{s}}{\partial t}-E_{s} \tan \psi_{s} \frac{\partial \psi_{s}}{\partial z} \\
& -\frac{n_{0}}{c} E_{s} \tan \psi_{s} \frac{\partial \psi_{s}}{\partial t}+\frac{\gamma_{s}}{2} E_{s}=\frac{g_{x}}{2} E_{s}+\frac{g_{x y}}{2} E_{s} \tan \psi_{s}
\end{aligned}
$$

When this equation is subtracted from Eq. (51) and the remainder is divided by $E_{s} \tan \psi_{s}$ the result can be written

$$
\begin{aligned}
\frac{\partial \psi_{s}}{\partial z} & +\frac{n_{0}}{c} \frac{\partial \psi_{s}}{\partial t}=-\frac{1}{2}\left[\left(g_{x}-g_{y}\right) \cos \psi_{s} \sin \psi_{s}\right. \\
& \left.+g_{x y}\left(\sin ^{2} \psi_{s}-\cos ^{2} \psi_{s}\right)\right] .
\end{aligned}
$$

In a similar manner, the equations for the pump field become

$$
\begin{aligned}
\frac{\partial E_{p}}{\partial z}+ & \frac{n_{0}}{c} \frac{\partial E_{p}}{\partial t}+\frac{\gamma_{p}}{2} E_{p} \\
= & -\frac{1}{2}\left[\left(\alpha_{x} \cos ^{2} \psi_{p}+2 \alpha_{x y} \cos \psi_{p} \sin \psi_{p}\right.\right. \\
& \left.\left.+\alpha_{y} \sin ^{2} \psi_{p}\right)\right] E_{p}, \\
\frac{\partial \psi_{p}}{\partial z}+ & \frac{n_{0}}{c} \frac{\partial \psi_{p}}{\partial t} \\
= & \frac{1}{2}\left[\left(\alpha_{x}-\alpha_{y}\right) \cos \psi_{p} \sin \psi_{p}+\alpha_{x y}\left(\sin ^{2} \psi_{p}-\cos ^{2} \psi_{p}\right)\right]
\end{aligned}
$$

Further simplifications can be made for the limit of an unsaturated quasi-steady-state laser amplifier in which pump depletion is not important. In this case, the gain coefficients become constants and $g_{x y}$ is zero when $n(\theta, \phi)$ is an even function of $\phi$, which is true for most practical situations. The field equations given in Eqs. (51) and (53) may be written under these conditions as

$$
\begin{aligned}
& \frac{d E_{s}}{d z}+\frac{\gamma_{s}}{2} E_{s}=\frac{1}{2} E_{s}\left(g_{x 0} \cos ^{2} \psi_{s}+g_{y 0} \sin ^{2} \psi_{s}\right), \\
& \frac{d \psi_{s}}{d z}=-\frac{1}{2}\left(g_{x 0}-g_{\mu 0}\right) \sin \psi_{s} \cos \psi_{s},
\end{aligned}
$$

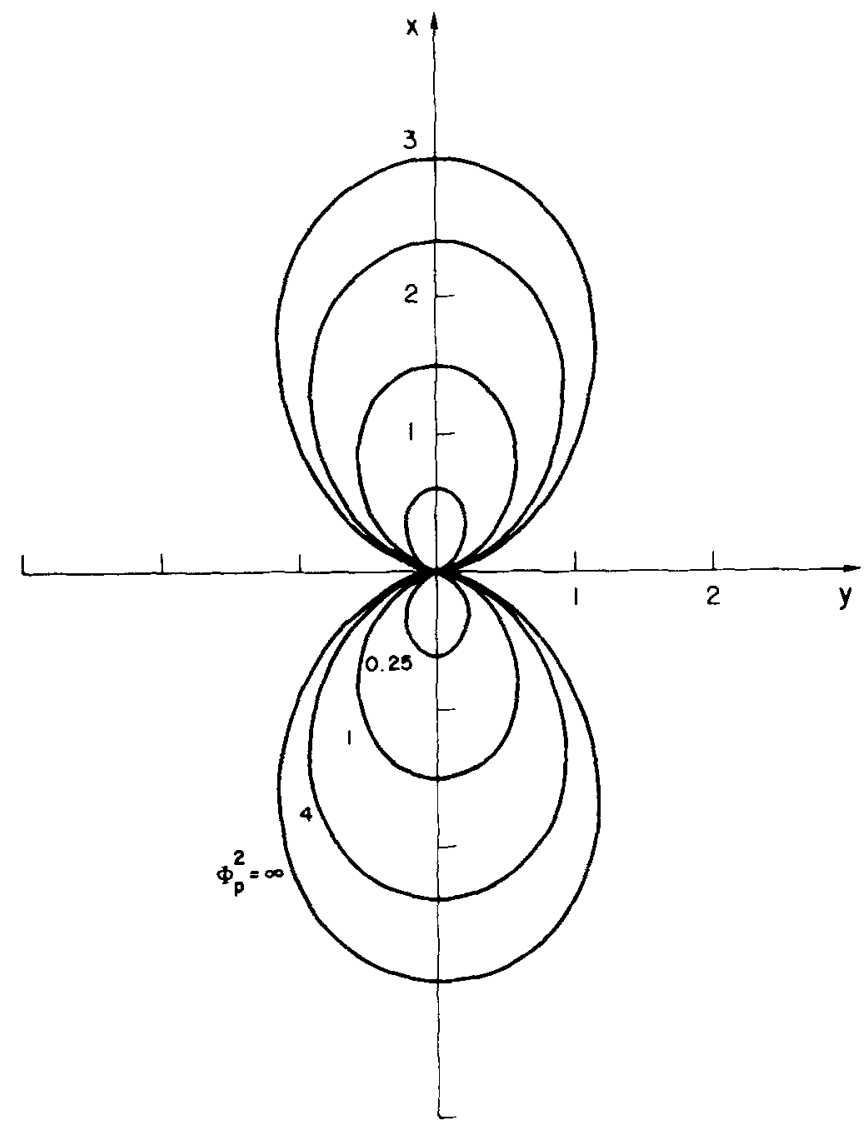

FIG. 4. Angular distribution of the unsaturated gain in a unidirectional ensemble of laser dipoles for various values of pump intensity. 
where $g_{x 0}$ and $g_{y 0}$ are the unsaturated gain coefficients. Equation (55b) can be solved for the $z$ dependence of the polarization angle $\psi_{s}$ and the result can be substituted into Eq. (55a) to solve for the field amplitude. The solution to Eq. (55b) is given by

$$
\tan \psi_{s}=\tan \psi_{s 0} \exp \left[-\left(g_{x 0}-g_{y 0}\right) z / 2\right],
$$

where $\psi_{s 0}$ is the initial polarization angle with respect to the $x$ axis. From this result it is clear that the polarization angle of the propagating signal tends to align with the $x$ axis $\left(\psi_{s}\right.$ $=0$ ) when $g_{x 0}$ is greater than $g_{y 0}$, and the polarization approaches the $y$ axis $\left(\psi_{s}=\pi / 2\right)$ when $g_{y 0}$ is greater than $g_{x 0}$.

Using Eq. (56), Eq. (55a) can be written

$$
\begin{gathered}
\frac{d \ln E_{s}}{d z}=-\frac{\gamma_{s}}{2}+\frac{1}{4}\left(\left(g_{x 0}+g_{y 0}\right)+\left(g_{x 0}-g_{y 0}\right)\right. \\
\left.\quad \times\left\{1+\tan ^{2} \psi_{s 0} \exp \left[-\left(g_{x 0}-g_{y 0}\right) z\right]\right\}^{-1 / 2}\right) .
\end{gathered}
$$

This equation can be integrated and the result is ${ }^{20}$

$$
\begin{aligned}
E_{s}= & E_{s 0} \exp \left\{-\frac{\gamma_{s} z}{2}+\frac{\left(g_{x 0}+g_{y 0}\right) z}{4}-\frac{1}{4} \ln \left[\left(\frac{1+\sec \psi_{s 0}}{1-\sec \psi_{s 0}}\right)\right.\right. \\
& \left.\left.\times\left(\frac{1-\left\{1+\tan ^{2} \psi_{s 0} \exp \left[-\left(g_{x 0}-g_{y 0}\right) z\right]\right\}^{1 / 2}}{1+\left\{1+\tan ^{2} \psi_{s 0} \exp \left[-\left(g_{x 0}-g_{y 0}\right) z\right]\right\}^{1 / 2}}\right)\right]\right\} .(58)
\end{aligned}
$$

Specific solutions can be obtained when the unsaturated gain terms are calculated for a particular molecular distribution.

Finally, Eq. (55a) can also be rewritten as

$$
\frac{d E_{s}}{d z}+\frac{\gamma_{s}}{2} E_{s}=\frac{l}{2} g_{0}\left(\psi_{s}\right) E_{s},
$$

where

$$
g_{0}\left(\psi_{s}\right)=g_{x 0} \cos ^{2} \psi_{s}+g_{y 0} \sin ^{2} \psi_{s},
$$

and can be considered an incremental gain distribution. $\mathbf{A}$ plot of $g_{0}\left(\psi_{s}\right)$ versus $\psi_{s}$ gives the incremental gain seen by a signal field for any polarization direction. These gain distributions are plotted for an unidirectional molecular distribution in Sec. IV.

\section{UNIDIRECTIONAL DISTRIBUTION}

The formulas that have been developed represent a complete set from which one may determine the polarization and amplitude characteristics of the propagating pump and signal fields in quite general optically pumped laser media. While these equations can always be solved by numerical methods, it turns out that certain special cases of the general solutions are sufficient to describe most situations of practical interest. In this section linearly polarized solutions are obtained in the case of a $\mathrm{cw}$ laser having a unidirectional molecular distribution. For convenience, the polarization of the pump field is taken to be parallel to the $\mathrm{x}$ axis and there is no pump depletion.

With a unidirectional distribution the function $n(\theta, \phi)$ can be written

$$
n(\theta, \phi)=N \delta\left(\theta-\theta_{0}, \phi-\phi_{0}\right),
$$

where $\theta_{0}$ and $\phi_{0}$ represent the preferred orientation and the $\delta$ function is normalized by

$$
\int_{\Omega} \delta\left(\theta-\theta_{0}, \phi-\phi_{0}\right) d \Omega=1 .
$$

Using these formulas Eqs. (44) become

$$
\begin{aligned}
& g_{x}(z)=N \sigma_{s}\left(v_{s 0}\right) D_{s}\left(\theta_{0}, \phi_{0}, z\right) \cos ^{2} \theta_{0}, \\
& g_{x y}(z)=N \sigma_{s}\left(v_{s 0}\right) D_{s}\left(\theta_{0}, \phi_{0}, z\right) \cos \theta_{0} \sin \theta_{0} \cos \phi_{0}, \\
& g_{y}(z)=N \sigma_{s}\left(v_{s 0}\right) D_{s}\left(\theta_{0}, \phi_{0}, z\right) \sin ^{2} \theta_{0} \cos ^{2} \phi_{0} .
\end{aligned}
$$

In this case, the gain coefficients have an explicit form and can be used in the field amplitude and phase equations directly. When $\theta_{0}$ and $\phi_{0}$ are both zero the molecules are all lined up parallel to the pump field and only the gain in the $x$ direction is nonzero. With Eqs. (32) and (33) this gain value is

$$
g_{x}(z)=3 N \bar{\sigma}_{s}\left(v_{s 0}\right) \Phi_{p x}^{2} /\left[1+\Phi_{p x}^{2}+\Phi_{s x}^{2}(z)\right]
$$

where $\bar{\sigma}_{s}$ is the average stimulated emission cross section equal to $\sigma_{s} / 3 .{ }^{21}$ This gain can be simplified to

$$
g_{x}(z)=g_{x 0} /\left[1+s I_{s x}(z)\right],
$$

where the unsaturated gain $g_{x 0}$ is given by

$$
g_{x 0}=3 N \bar{\sigma}_{s}\left(v_{s 0}\right) \Phi_{p x}^{2} /\left(1+\Phi_{p x}^{2}\right)
$$

and the saturation parameter $s$ is

$$
s=\left[\tau_{3} \sigma_{s}\left(v_{s 0}\right) / h v_{\mathrm{so}}\right]\left[1 /\left(1+\Phi_{p x}^{2}\right)\right] .
$$

The unsaturated gain distribution from Eq. (60) in this case is

$$
g_{0}\left(\psi_{s}\right)=N \bar{\sigma}_{s}\left[3 \Phi_{p x}^{2} /\left(1+\Phi_{p x}^{2}\right)\right] \cos ^{2} \psi_{s} .
$$

This distribution can be normalized by $N \bar{\sigma}_{s}$, and the dimensionless unsaturated gain distribution is shown in Fig. 4 for several different values of $\Phi_{p x}^{2}$. It can be seen that no matter how intense the pump field is, there will be very little gain for signal fields polarized in a direction nearly parallel to $y$. This result can also be seen from the field equations. Using Eq. (65) and Eqs. (34) and (35) to convert to intensities, the field equations become

$$
\begin{aligned}
& \frac{d I_{s x}}{d z}=\frac{g_{x 0} I_{s x}}{1+s I_{s x}}-\gamma_{s} I_{s x}, \\
& \frac{d I_{s y}}{d z}=-\gamma_{s} I_{s y} .
\end{aligned}
$$

Equation (69a) is a standard laser amplifier equation and its solutions are well known. The $y$ component from Eq. (69b) experiences no gain due to the unidirectional distribution and is actually attenuated due to the loss mechanisms.

Finally, it may be noted that if the laser is unsaturated and tuned to line center, the signal polarization direction and amplitude are given explicitly by Eqs. (56) and (58) with $g_{x 0}=0$. Thus the signal tends to align itself parallel to the molecular orientation. On the other hand, one can show from Eq. (54b) that the pump field tends to align itself perpendicular to the molecules as the parallel pump component is preferentially absorbed. Other solutions for the unidirectional distribution can also be readily obtained.

\section{CONCLUSION}

In this paper a semiclassical formalism has been developed for investigating the amplitude and polarization char- 
acteristics of the pump and signal fields propagating in a dyelaser amplifier. In their most general form these results allow for arbitrary amplitudes and elliptical polarizations of the fields which may also be tuned away from the center frequencies of the absorption and emission transitions. To obtain detailed solutions of the governing equations, numerical techniques are often required. However, for certain important limits the equations simplify and analytic solutions become possible. Particularly helpful simplifications occur for lasers tuned near line center. In this case the pump and signal fields can remain linearly polarized, and some exact solutions have been described. Emphasis has been placed on unidirectional molecular distributions so that the gain has a strongly preferred direction. As one should expect, the signal polarization tends to become aligned with the gain maximum. For an isotropic molecular distribution the results are somewhat more complicated, and several solutions and applications are discussed in the following study. ${ }^{12}$

'P. P. Sorokin, J. R. Lankard, E. C. Hammond, and V. L. Moruzzi, IBM J. Res. Dev. 11, 130 (1967).

${ }^{2}$ B. I. Stepanov and V. P. Gribkovskii, Theory of Luminescence (Iliffe Books, London, 1968).
${ }^{3}$ P. P. Feofilov, The Physical Basis for Polarized Emission (Consultants Bureau Enterprises, New York, 1961), p. 2.

${ }^{4}$ K. H. Drexhage, in Topics in Applied Physics: Dye Lasers, Vol. 1, edited by F. P. Schaefer (Springer-Verlag, New York, 1973), p. 144.

'F. P. Schaefer, in Topics in Applied Physics: Dye Lasers, Vol. 1, edited by F. P. Schaefer (Springer-Verlag, New York, 1973), p. 7.

${ }^{6}$ P. P. Feofilov, Ref. 3, p. 127.

${ }^{7}$ L. L. Chapoy and D. B. DuPre, J. Chem. Phys. 69, 519 (1978).

${ }^{8} \mathrm{G}$. Weber, in Fluorescence and Phosphorescence Analysis, edited by D. M. Hercules (Interscience, New York, 1966), p. 217.

${ }^{9}$ A. N. Sevchenko, A. A. Kovalev, V. A. Pilipovich, and Yu. V. Razvin, Sov. Phys. Dok. 13, 226 (1968)

${ }^{10}$ A. Lempicki, Acta Phys. Pol. A 50, 179 (1976).

"D. W. Phillian, D. J. Kuizenga, and A. E. Siegman, J. Chem. Phys. 61, 3828 (1974).

${ }^{12}$ K. C. Reyzer and L. W. Casperson, J. Appl. Phys. 51, 6083 (1980).

${ }^{13}$ K. C. Reyzer and L. W. Casperson (unpublished).

${ }^{14} \mathrm{H}$. Haken, in Handbook of Physics, edited by L. Genzel (Springer-Verlag, New York, 1970), p. 208.

${ }^{15}$ W. H. Louisell, Radiation and Noise in Quantum Electronics (McGrawHill, New York, 1964), p. 223.

${ }^{16}$ A. Yariv, Quantum Electronics (Wiley, New York, 1975), p. 150.

${ }^{17}$ B. B. Snavely, in Topics in Applied Physics: Dye Lasers, Vol. 1., edited by F. P. Schaefer (Springer-Verlag, New York, 1973), p. 89.

${ }^{16}$ I. Nagata and T. Nakaya, J. Phys. D 6, 1870 (1973).

${ }^{19}$ B. B. Snavely, Ref. 17, p. 89-90.

${ }^{20}$ I. S. Gradshteyn and I. W. Ryzhik, Tables of Integrals, Series and Products (Academic, San Francisco, 1965), Eq. 2.315.

${ }^{21} \mathrm{G}$. Mourou and M. M. Denariez-Roberge, IEEE J. Quantum Electron. QE9, 787 (1973). 